\title{
IL CAMBIAMENTO RADICALE DELLE POLITICHE MIGRATORIE: DAL LASCIAR VIVERE AL LASCIARE MORIRE (DALLA BIOPOLITICA A SEMPRE PIÙ TANATOPOLITICA)
}

\author{
The Radical Change in Migration Policies: from Letting Live \\ to Letting Die (from Biopolitics to More and More Thanatopolitics)
}

Salvatore Palidda*

\begin{abstract}
Riassunto. Il testo descrive gli aspetti salienti dell'attuale congiuntura mondiale. Attraverso una sintetica analisi del processo che ha portato al trionfo del liberismo globalizzato, si mostra come a cominciare dal 1990 la guerra alle migrazioni si inscriva in una vera e propria tanatopolitica (il lasciar morire). Infatti, i dominanti designano le migrazioni come il nemico del $X X I^{\circ}$ secolo perché temono che l'aumento della popolazione mondiale sia incontrollabile e che si sovrapponga ai cambiamenti climatici provocando secondo loro - destabilizzanti invasioni di migranti nei paesi ricchi. In realtà temono che si imponga la necessità di una redistribuzione egualitaria della ricchezza mondiale che potrebbe permettere la sopravvivenza decente anche di oltre 10 miliardi di umani a condizione anche di eliminare tutte le fonti di distruzione del pianeta e innanzitutto l'estrattivismo di carbone, petroli, gas, uranio e terre rare.
\end{abstract}

Parole chiave: tanatopolitica delle migrazioni; liberismo globalizzato; migrazioni disperate; guerra alle migrazioni; guerre climatiche.

\begin{abstract}
The text describes the salient aspects of the current world situation. Through a synthetic analysis of the process that led to the triumph of globalized liberalism, it is shown how starting in 1990 the war on migration is inscribed in a real thanatopolitics (letting die). In fact, the dominant people designate migration as the enemy of the 21st century because they fear that the increase in the world population is uncontrollable and that it overlaps with climate change causing according to them - destabilizing invasions of migrants in rich countries. In reality, they fear that there is a need for an egalitarian redistribution of world wealth that could allow the decent survival of over 10 billion humans also on the condition of eliminating all sources of destruction on the planet and above all gas, uranium and rare earths.
\end{abstract}

Keywords: migration thanatopolitic; globalized liberalism; desperate migrations; war on migration; climate wars.

Facoltà di Scienze della Formazione dell'Università degli Studi di Genova. Genova, Italia. E-mail: palidda@unige.it. Orcid: https://orcid.org/0000-0002-9644-9405. 
Come sempre, i disastri colpiscono molto di più le persone che sono prive di protezione e anzi sono oggetto di persecuzioni da parte delle polizie, anche perché non hanno i mezzi e le conoscenze necessarie per proteggersi. Che si tratti di guerre, terremoti, tsunami, disastri industriali e di pandemie, il rischio che la stragrande maggioranza delle vittime sia fra i più poveri e meno protetti è sempre certo. Fra il quasi mezzo milione di morti negli Stati Uniti a causa dell'attuale pandemia Sars Covid19 (a fine febbraio 2021) ci sono ben poche o rarissime persone come Trump e i suoi pretoriani o come Bolsonaro o Macron e altri dominanti. La maggioranza delle vittime è sempre costituita da quella parte della popolazione costantemente inferiorizzata, razzializzata, considerata "umanità a perdere" o "umanità in eccesso" o "umanità ormai inutile" (come è notoriamente il caso degli anziani nelle case di riposo che sono diventati covi di pandemia all'abbandono).

Come vedremo dopo i casi più drammatici - non a caso - sono quelli alle frontiere dell'Europa, dove la guerra alle migrazioni si materializza sino all'estremo di brutalità e di cinismo; lo stesso vale negli Emirati Arabi e in Arabia Saudita dove gli immigrati privati di passaporto, rimasti senza lavoro per la pandemia, non possono rientrare a casa e non hanno più né di che mangiare né dove stare. Questa "modalità" di governo dell'"umanità a perdere" si chiama tanatopolitica (il lasciar morire) e vedremo perché oggi rischia di affermarsi come la modalità prevalente rispetto anche alla tradizionale biopolitica (il lasciar vivere per meglio sfruttare).

\section{I più importanti cambiamenti nelle migrazioni e i confini dopo la "rivoluzione" liberista globalizzata}

La storia delle migrazioni è segnata da periodi di razzismo e rifiuto violento e anche da periodi di integrazione e assimilazione pacifica nei paesi di arrivo (ma sempre a costi morali e materiali molto alti). Lo sviluppo capitalista del diciannovesimo e ventesimo secolo fu alimentato dalla migrazione sia interna che internazionale.

Ma, dall'inizio del 1990, la migrazione è diventata lo spettro che sembra terrorizzare i paesi ricchi come se fosse una minaccia particolarmente pericolosa (anche perché buona parte dei media e dei vertici militari e delle polizie fa l'amalgama col terrorismo). La definizione delle migrazioni come minaccia (quindi un termine prettamente militare) di fatto è diventata legittimazione della guerra permanente contro le migrazioni. Questo giustifica la continua proliferazione di mezzi e forze militari e di polizia destinati a tale scopo, nonché una serie di sofisticati dispositivi tecnologici e il dispendio di enormi risorse economiche. 
Dopo la caduta del muro di Berlino nel 1989, la proliferazione dei muri è stata spaventosa ${ }^{1}$. Quando avvenne questa caduta del Berliner Mauer si contavano nel mondo 15 barriere fisiche, una decina in più rispetto a quante ne esistevano alla fine della Seconda guerra mondiale: oggi queste sono 70 , con altre 7 già finanziate e in via di completamento ${ }^{2}$.

Quella attribuita alle migrazioni è diventata una delle minacce più gravi del ventunesimo secolo. Sta qui il cambio di paradigma nella comprensione e nel trattamento delle migrazioni e dei confini, provocando disperazione, tragedie e morti tra i migranti.

Questo cambiamento nel paradigma delle migrazioni e dei confini si osserva nella letteratura che ha subito un enorme sviluppo negli ultimi 20 anni.

Diversi autori hanno descritto i vari aspetti delle attuali drammatiche situazioni migratorie. Tuttavia, gran parte di queste letture è parziale e costruita come una reiterazione di lavori precedenti; le narrazioni seguono spesso un modello di discorso dominante basato sulla "scienza delle migrazioni" del diciannovesimo e ventesimo secolo, priva di una sua decostruzione e messa in discussione (come insegnano Foucault e Sayad ${ }^{3}$ ).

Questo cambiamento nel paradigma della migrazione e dei confini spesso non è riconosciuto dal mondo accademico a causa di due gravi lacune. In primo luogo, c'è stata troppa poca attenzione dedicata alla comprensione delle conseguenze della "rivoluzione" neoliberista globalizzata, iniziata negli anni '70 nel Nord del mondo (fanno eccezione il lavoro di Sassen, 1999, 2008, 2014 e anche di altri). La seconda lacuna è la mancanza di analisi della percezione tra i dominanti della cosiddetta crescita incontrollata della popolazione mondiale (specialmente nei paesi più poveri del Sud del mondo) e della sua presunta sovrapposizione ai cambiamenti climatici. Questa sovrapposizione suscita nelle élite il terrore che possa essere generatrice di "invasioni pericolose" di migranti poveri nei paesi ricchi (Miller, 2017; Palidda, 2019). Questo è il pauroso "spettro" che preoccupa le élite dei paesi dominanti ed è la ragione principale del passaggio da una biopolitica delle migrazioni favorevole all'integrazione dei migranti nella società di arrivo (per meglio sfruttarli) alla tanatopolitica che è lasciar morire (tesi che rimanda al lavoro di Michel Foucault, 2004 e 2005; vedi anche Aradau e Tazzioli, 2020). Le migrazioni e le frontiere si spostano e i migranti diventano "vite di scarto o spazzatura" (Bauman, 2003) o rimangono intrappolati come "umanità in eccesso" destinata a essere smaltita come rifiuti ingombranti o tossici.

\footnotetext{
Christian Elia. Oltre i muri. Storie di comunità divise. Milieu Edizioni, 2019.

Disponibile su: <https:/www.fanpage.it/attualita/tutti-i-muri-nel-mondo-30-anni-dopo-berlino/> .

3 Per la decostruzione del discorso dominante si veda innanzitutto l'opera di Foucault e per gli studi sulle migrazioni si veda la critica di Sayad (2002) alla cosiddetta "scienza delle migrazioni".
} 


\section{Migrazione fatto politico totale del XXI secolo}

Per comprendere appieno un tale cambio di paradigma, occorre guardare ciò che effettivamente è mutato nelle migrazioni e nelle frontiere, nel contesto che s'è sviluppato in particolare dal 1990 con lo sviluppo del neoliberismo globalizzato. Oggi più che mai le migrazioni e le frontiere sono fatti politici totali (reinterpreto qui il concetto classico di Marcel Mauss ${ }^{4}$ ). Fatti politici totali non solo perché sempre al centro delle preoccupazioni politiche anche se talvolta come tema correlato a quella della sicurezza, del terrorismo e persino della criminalità organizzata. Ma anche perché scuote profondamente l'immaginario etico e morale e quindi culturale di tutti i paesi perché diventati tutti di emigrazione, di immigrazione e di transito. Non è infatti casuale che ci sia un revival del colonialismo che è appunto neocolonialismo piuttosto e post-colonialismo; quest'ultima accezione presenta infatti alcune ambiguità e un approccio culturalista che di fatto ignorano la realtà nuda e cruda del neocolonialismo in tutte le sue dimensioni economiche, sociali, politiche e culturali e in particolare la riproduzione di neo-schiavitù e della violenza estrema che le impone producendo nessi inestricabili fra razzializzazione, sessismo e neofascismo.

La biopolitica delle migrazioni mirava a integrare i "buoni" immigrati insieme al periodico rigetto, la razzializzazione e i tentativi di eliminare i "cattivi immigrati" (Palidda, 2018a, 2018b). Gli immigrati ancora oggi sono - in parte - tollerati se si relegano alla condizione di inferiorizzazione, come "scimmie ammaestrate" o servi a disposizione dei dominanti ${ }^{5}$. L'integrazione e ancor di più l'assimilazione li trasforma spesso in lavoratori docili e talvolta in buoni cittadini che pagano le tasse, e che sono anche pronti a morire nelle guerre del nuovo paese della loro integrazione/assimilazione. I casi degli Stati Uniti e della Francia sono esemplari di tali pratiche classiche di integrazione e assimilazione degli immigrati. La migrazione è sempre stata essenziale per lo sviluppo economico di questi paesi, che grazie anche al super sfruttamento degli inferiorizzati sono diventati potenze economiche, politiche e militari del mondo. Ed è grazie al continuo arrivo di flussi migratori che gli Stati Uniti e altri paesi hanno avuto una grande crescita economica dal 1990 e hanno superato la crisi del 2008. La popolazione degli Stati Uniti è infatti passata dai 250 milioni del 1990 a quasi 340 milioni nel 2021, oltre a 11 milioni di immigrati privi di documenti soggetti al super sfruttamento e alle neo-schiavitù. Le migrazioni hanno quindi avuto un ruolo utilitaristico chiave: questo paradigma migratorio è stato funzionale allo sviluppo della società industriale governata dagli stati nazionali sovrani sino agli

\footnotetext{
4 Marcel Mauss (nipote di Durkheim) fu uno dei celebri padri dell'antropologia; è a lui che si deve la revisione del concetto di "fatto sociale" proposto da questo suo zio con "fatto sociale totale" che include tutti gli aspetti di ogni fenomeno. Penso che oggi sia fondamentale rivisitare questo concetto come "fatto politico totale" poiché tutti gli aspetti coinvolti contengono una forte significato politico.

5 Vedi: <http://remhu.csem.org.br/index.php/remhu/article/view/523>.
} 
anni '70. Si sfrutta infatti l'aspirazione all'emancipazione degli emigrati-immigrati che si combina spesso con lo "spirito del capitalismo" e quindi del liberismo. Così l'etnicizzazione dei migranti permette a caporali e power-brokers etnici di sfruttare ancora di più i propri connazionali così come facevano le mafie delle varie nazionalità negli Stati Uniti degli anni Venti e ancora dopo.

\section{Alle origini del cambiamento}

Le origini del cambiamento del paradigma migratorio classico sono emerse dagli anni '70 in poi con l'accelerazione della rivoluzione neoliberista globale.

Lo stop delle migrazioni per lavoro fu adottato dai paesi dell'OCSE (Organizzazione per la cooperazione e lo sviluppo economico) nel 1974, dopo la crisi petrolifera del 1973. Ma l'immigrazione è continuata negli anni '70 e '80, soprattutto dopo la fine del regime di Salazar in Portogallo e di quello di Franco in Spagna. Dalla metà degli anni '80 I'Italia e la Spagna sono diventati paesi di immigrazione ma in realtà quasi tutti i paesi del mondo, sia al loro interno sia con I'estero, sono diventati paesi di emigrazioni, immigrazioni o anche di transito. Le migrazioni sono proliferate anche a seguito delle scelte delle dittature in vari paesi dell'America Latina, Africa e Asia e poi soprattutto per il galoppante degrado delle condizioni di lavoro e di vita. Da allora si intensificano le migrazioni dal Messico e dall'America Centrale verso gli Stati Uniti così come quelle tra i Sud poveri e i Sud ricchi fra i quali emergono gli Emirati e l'Arabia Saudita. Le delocalizzazioni dai paesi ricchi verso il cosiddetto Terzo Mondo non hanno affatto migliorato la situazione: hanno sempre portato in questi paesi condizioni di lavoro invivibili, salari miserabili ma anche tante morti sul lavoro (si ricordino i grandi disastri in Bangladesh, in Sri-Lanka e in tanti altri paesi oltre che l'inquinamento spaventoso dappertutto in Asia, Africa e America Latina).

Negli anni '70 esistevano casi di accoglienza dei rifugiati sebbene per motivi politici strumentali. Ricordiamo il fenomeno dei boat people dal sud-est asiatico dopo la fine della guerra del Vietnam e le conseguenze del regime di Pol Pot in Cambogia. Dopo il periodo della Guerra Fredda, ci fu anche la successiva migrazione di massa dai paesi dell'Europa orientale, cioè dopo il crollo dell'URSS nel 1991. Ma dal 1990 comincia l'escalation del rigetto degli immigrati insieme al degrado crescente delle condizioni di lavoro e di vita di quelli che riescono ad arrivare nei paesi ricchi.

In realtà dal 1990 al 2020 lo stop ufficiale delle migrazioni, la carcerizzazione di una parte degli immigrati senza documenti, i respingimenti e le espulsioni (Squire, 2016; Palidda, 2009) si combinano con la faccia ancora più sporca delle pratiche del governo delle migrazioni: la regolazione tacita dell'immigrazione irregolare per disporre dei neo-schiavi. In tutti i paesi le polizie usano sia la gestione violenta, sia la gestione informale di lasciar correre: negli Stati Uniti come nei principali paesi dell'Europa dell'Ovest (Regno Unito, Francia, Germania, Italia, 
Spagna ecc.) la prassi prevalente è che si tollera il lavoro nero, la neo-schiavitù, le condizioni di vita indigenti degli immigrati senza documenti e allo stesso tempo la pronta repressione violenta di chi osa ribellarsi a tali condizioni e reclama l'integrazione regolare e pieni diritti ${ }^{6}$. In altre parole, la tecnica del governo dell'immigrazione in tutti i paesi ricchi è di creare una massa di manodopera "usa e getta" che viene costantemente rinnovata con nuovi arrivi detti "clandestini" ma in realtà ben noti alle polizie, che li gestiscono spesso insieme a caporali e vari brokers esperti nel camuffare l'irregolarità. E tale sistema funziona anche per una parte dei lavoratori autoctoni e anche nelle grandi imprese e nel cuore stesso delle grandi città (vedi nota 7).

Così i migranti sono costretti all'irregolarità dalla partenza dai loro paesi, lungo i tragitti spesso tragici per via marittima o per via terrestre e poi nel paese di approdo sin quando non vengono di nuovo espulsi come "rifiuti umani". Le peripezie e le morti di queste persone non sono da meno di quelle dei disperati che scappavano dalle persecuzioni e violenze dei regimi fascisti e nazisti o dei superstiti che scappavano dal fronte nella prima e nella Seconda guerra mondiale o che facevano parte della Resistenza antifascista.

Come sempre il proibizionismo provoca morte: dalla fine degli anni ' 80 , decine di migliaia di migranti sono morti durante i tentativi di migrazione per raggiungere i paesi ricchi (soprattutto ai confini degli Stati Uniti e dell'Europa mediterranea).

Tutto ciò è avvenuto a seguito dello smantellamento di gran parte dell'apparato industriale nei paesi più sviluppati nel nord del mondo, e quindi il suo trasferimento nel sud del mondo a bassi salari, in paesi meno regolamentati (con delocalizzazioni che hanno incluso anche le attività terziarie come i call center). I paesi ricchi hanno così sperimentato una minore necessità di lavoro di immigrati da integrare/assimilare nella produzione stabile, e hanno optato sempre più per il solo lavoro precario e/o neo-schiavizzato nelle economie sommerse (che oggi si possono osservare nel cuore stesso delle grandi metropoli occidentali come Londra, Parigi, Los Angeles e New York ecc.). Una tale trasformazione epocale è stata resa possibile con la rivoluzione tecnologica nelle comunicazioni, nei trasporti e nella produzione e anche dalla rivoluzione finanziaria (che impoverisce ulteriormente il Sud del mondo).

La rivoluzione tecnologica ha innescato anche i cambiamenti negli affari militari e di polizia che si traducono in mutamento politico, cioè dei rapporti di forza fra dominanti e dominati. Lo sfruttamento delle nuove tecnologie e dei dispositivi di sorveglianza e coercizione ha fatto sì che polizie e militari (anche privati) si sovrappongono e collaborano sempre più in nome della sicurezza totale che diventa continuum delle guerre permanenti su scala globale e guerra

\footnotetext{
${ }_{6}$ Vedi: <http://remhu.csem.org.br/index.php/remhu/article/view/523> e anche <http://www.
} socioscapes.org/index.php/sc/article/download/14/3/>. 
sicuritaria su scala nazionale e locale contro i migranti, rom, marginali e oppositori delle scelte liberiste. Questo processo ha generato una maggiore asimmetria fra dominanti e dominati sempre più privi di possibilità di protezione. Non è casuale che negli ultimi 40 anni la concentrazione di capitali e mezzi da parte della piccola minoranza di ricchi è stata sempre maggiore mentre allo stesso tempo i meno abbienti e gli svantaggiati sono diventati sempre più poveri e sempre più privi di protezioni. Lo scenario così polarizzato ha rafforzato la crescente asimmetria di potere. Ciò consente alle élite dei paesi dominanti di imporre ai dominati sempre peggiori condizioni di lavoro, redditi più bassi e cattive condizioni di vita, ciò in particolare a gran parte delle popolazioni dei paesi in via di sviluppo o più poveri. Queste pratiche di dominio e di super sfruttamento si trovano nelle economie sommerse che sono spesso attività per neo-schiavi sottoposti con la violenza a tale condizione (fra i casi emblematici si veda quanto avviene con le ditte del subappalto anche nelle grandi aziende statali e para-statali ${ }^{7}$ ).

La revolution in military affairs e in police affairs e di polizie e militari dello stato o anche privati oltre che di mafie e caporali sono lo strumento coercitivo che impone e garantisce questo ordine sociale di drastica disuguaglianza su scala mondiale, nazionale e locale.

Un altro fattore che spiega questa trasformazione globale è l'intensificazione delle pratiche neocoloniali attraverso l'eccessivo sfruttamento delle risorse e la devastazione dei territori nel Sud del mondo. Questo processo include la depredazione di grandi territori, le espulsioni delle popolazioni che vi vivono (vedi in Asia, in America Latina e anche in Africa) per opera delle multinazionali e a sua volta l'aggravarsi della crisi ecologico-politica globale. Di conseguenza, tale processo provoca nuove migrazioni disperate di persone che fuggono da territori devastati dove nemmeno un filo d'erba cresce, né i pesci sopravvivono o possono essere pescati con i metodi tradizionali (si pensi fra altri al caso del Senegal dove le grandi navi da pesca fanno razzia della fauna locale lasciando in miseria i pescatori locali). La migrazione sussume sempre più le conseguenze di disastri economici, ecologici sociali, culturali e dunque politici provocati soprattutto nei paesi del Sud dalle lobby dei paesi dominanti e dalle loro multinazionali e istituzioni finanziarie (Palidda, 2018a).

\footnotetext{
Vedi: < http://effimera.org/il-furore-di-sfruttare-e-di-accumulare-di-gianni-giovannelli-e-turi-palidda/> ; Super sfruttamento e corruzione nel subappalto al cuore del sistema di produzione Fincantieri: <http://www.labottegadelbarbieri.org/modello-genova-ma-per-favore/>; $\quad<$ https://blogs.media part.fr/salvatore-palidda/blog/040320/immigres-hyper-exploites-dans-la-construction-du-siege-dumonde-et-leur-revolte> ; non mancano i casi di guardiani armati a Canary Wharf, uno dei quartieri degli affari più importanti di Londra con patentino comprato sottobanco: <www.bbc.com/news/ av/uk-england-london-32008983/security-guard-fraud-exposed-by-undercover-researchers > ; $<$ www.bbc.com/news/uk-england-london-31876590 > così come sempre più casi di corruzione di funzionari e agenti delle polizie.
} 
Queste conseguenze della rivoluzione economica neoliberale globale generano i nuovi disastri umanitari e le vere minacce alla sicurezza umana descritte da Gros (2008).

Ma va ricordato che la maggior parte delle migrazioni avviene oggi tra i paesi poveri del Sud del mondo e i paesi ricchi (come gli Emirati, I'Arabia Saudita dove il gli immigrati sono in casi estremi di schiavitù e "vite da scarto").

I migranti sanno bene che rischiano di morire lungo il tragitto e che se approderanno da qualche parte probabilmente troveranno condizioni infelici ma forse potranno cavarsela e comunque restare da dove si parte è impossibile. Forse non è esagerato dire che il neocolonialismo di oggi è spesso peggiore di quello conosciuto nel $X I X^{\circ}$ secolo e ancora nel $X X^{\circ}$. II degrado dei territori e delle condizioni di vita in tutte le zone di emigrazione è spaventoso e appunto spinge a emigrazioni disperate. E' esattamente la conseguenza più brutale e quasi genocida dell'arricchimento continuo e sfrenato delle multinazionali e lobby.

La rivoluzione finanziaria ha facilitato I'ascesa della speculazione in borsa e dei paradisi fiscali. Gli stati-nazione hanno perso il controllo di queste manipolazioni finanziarie (in parte a causa dell'imperativo neoliberista della deregolamentazione), e quindi la transnazionalizzazione finanziaria è aumentata. Questo processo ha permesso anche l'ascesa globale della potente troika finanziaria (cioè Banca Mondiale, Organizzazione Mondiale del Commercio e Fondo Monetario Internazionale - FMI). Inoltre, la troika europea (ossia la Commissione europea, la Banca Centrale Europea e il FMI) così come il potere delle banche multinazionali private dei paesi ricchi dominanti (Stati Uniti, Cina, Europa e Giappone) svolgono tutti un ruolo chiave nella configurazione economica del neoliberismo globale. L'impatto del potente sistema finanziario globale ha causato un impoverimento ancora maggiore dei paesi meno sviluppati, in particolare una distribuzione sempre più diseguale della ricchezza e del reddito, e I'aumento della povertà.

Lo sviluppo del dominio economico liberista è di tipo transnazionale e si impone a sprezzo di tutto e di tutti. La sottomissione dei paesi deboli passa anche tramite la speculazione finanziaria (si veda "colpi di stato finanziari", Perkins, 2005).

A partire dagli anni '90, lo sviluppo del neocolonialismo economico neoliberista globalizzato è diventato sempre più sfrenato e da allora provoca sempre più disastri in parte persino per gli stessi paesi ricchi (si pensi all'aumento di uragani e tsunami notoriamente dovuto ai cambiamenti climatici provocati dall'aumento dell'inquinamento alimentato da consumo di carbone, petrolio, gas e uranio cioè dall'estrattivismo). La mentalità alla base di questo fenomeno è illustrata nei famigerati commenti del 1991 di Lawrence Summers, allora capo del dipartimento economico della Banca Mondiale (in seguito Segretario al Tesoro degli Stati Uniti), che scrisse in una nota privata trapelata alla stampa: 
I paesi africani sono in gran parte scarsamente inquinati. ... Dobbiamo incoraggiare una migrazione significativa dalle industrie inquinanti verso i paesi meno sviluppati ... la logica economica richiede che le masse di rifiuti tossici vengano gettate dove i salari sono bassi ed è indiscutibile. (Estratti pubblicati da The Economist (2 agosto 1992) e The Financial Times (2 ottobre 1992) con il titolo "Salvate il pianeta degli economisti")

E aggiunge, sempre nel 1991:

Il rischio di un'apocalisse a causa del riscaldamento globale o di qualsiasi altra causa è inesistente. L'idea che dovremmo imporre limiti alla crescita a causa di limiti naturali è un errore profondo; inoltre è un'idea il cui costo sociale sarebbe stupefacente se fosse applicata. ${ }^{8}$

Da allora, le "migrazioni disperate" sono aumentate in modo esponenziale. E' allora sbagliato parlare di "migrazioni climatiche", perché non è il cambiamento del clima in sé che genera questo: si tratta di migrazioni che inglobano tutti i disastri provocati dall'impatto delle multinazionali dei paesi dominanti nel Sud del mondo, appunto il fatto politico totale del XXI ${ }^{\circ}$ secolo (vedi Palidda, 2018b).

$L^{\prime}$ élite dei paesi dominanti sanno che le migrazioni disperate sono e saranno sempre più la conseguenza delle attività delle multinazionali nei paesi terzi e la conseguenza del loro accaparramento della ricchezza mondiale e dell'accesso alle innovazioni tecnologiche e alle scoperte scientifiche. Sanno che si potrebbe permettere di far vivere decentemente sul pianeta 8 o anche 10 miliardi di esseri umani, a condizione di eliminare l'estrattivismo (di carbone, petrolio, gas, uranio e "terre rare") e tutte le fonti e cause di disastri sanitari e ambientali che devastano sempre più enormi territori e la loro continua accumulazione di capitali. Non a caso tanti ultramiliardari del mondo si professano filantropi, benefattori dei poveri facendo dire ai media che regalano anche qualche miliardo. Nascondono così che questi capitali sono il prodotto di neo-schiavitù in particolare nelle delocalizzazioni delle loro attività sparse attraverso i paesi poveri o persino su navi nelle acque internazionali. E non è neanche casuale che per scongiurare la minaccia migratoria del $\mathrm{XXI}^{\circ}$ secolo alcuni militari, geo-ingegneri ed esperti di nuove tecnologie pretendono ideare anche le "guerre climatiche", che ovviamente avrebbero come obiettivo i paesi di emigrazione ${ }^{9}$. Fortunatamente queste ipotesi sembrano - per ora - non realizzabili o a rischio di boomerang. Ma è un errore ignorarle perché è di questi teoremi che si nutrono complottisti, negazionisti, razzisti e fascisti vari.

${ }_{8}$ Lawrence Summers, in occasione dell'Assemblea annuale della Banca Mondiale e del FMI a Bangkok nel 1991, intervista con Kirsten Garrett, "Background Briefing", Australian Broadcasting Company, secondo programma.

9 Vedi qui: <http://effimera.org/negazionismo-scetticismo-o-resistenze-dove-va-lecologia-politicadi-turi-palidda/ $>$. 


\section{La violenza pandemica globale}

L'attuale pandemia di Covid-19 rischia di essere una piaga ancora più grave per i paesi poveri, cioè i paesi di emigrazione. Le conseguenze contemporanee della pandemia Covid-19 per i migranti e le zone di confine, evidenziano in modo drammatico le caratteristiche chiave del cambio di paradigma.

Tra le prime misure adottate da quasi tutti i governi nella crisi del Sars Covid-19 c'è stata la chiusura delle frontiere (o la restrizione su larga scala della migrazione) e l'abbandono di ogni dovere di aiutare i rifugiati, che ha provocato il tragico aggravamento della situazione dei migranti situati vicino ai confini, soprattutto nella zona di confine turco-greca, nei confini nei Balcani, in quello spagnolo in Marocco (Ceuta e Melilla).

Col capitalismo globale, le pandemie circolano a livello internazionale con facilità e prosperano non solo nelle grandi metropoli, ma si diffondono anche in regioni scarsamente popolate. Prima dell'attuale pandemia negli ultimi 20 anni ci sono state molte altre epidemie: Ebola, SARS, influenza aviaria e suina. La mappatura globale dell'origine delle pandemie è esattamente al centro della produzione capitalistica mondiale. Tuttavia, ci sono molti altri luoghi in cui i rischi ambientali di mutazione dei virus e di diffusione sono più elevati. La "morte nera", o peste, iniziò in Mongolia, la "spagnola" del 1918 si estendeva oltre i confini nazionali dalla Francia, si pensa che I'HIV/AIDS abbia avuto origine in Africa così come il virus del Nilo occidentale e dell'Ebola, mentre la dengue sembra fiorire in America Latina. Le origini delle pandemie sono diverse, ma possiamo vedere l'impatto economico e demografico dei virus diffusi oltre i confini, facilitato dalla crescente globalizzazione nel corso degli anni. Nel caso della pandemia da Covid-19, molti la chiamano una "vendetta" del sistema estrattivista neoliberista violento, globale e deregolamentato. Alcune recenti ricerche hanno dimostrato che c'è una netta correlazione fra inquinamento e diffusione del Sars-Covid19 (così come in generale per le malattie cardiovascolari e quelle di raffreddamento, cioè le influenze stagionali, come concause). In tutti i paesi, una delle prime conseguenze della pandemia è stata l'aggravamento delle condizioni di lavoro e di vita delle persone più deboli e meno protette: lavoratori irregolari, senzatetto, lavoratori precari, detenuti, donne migranti che si prendono cura della popolazione vulnerabile (come collaboratori domestici e badanti) e, in generale, persone con redditi incerti e bassi. È emblematico, ad esempio, che la maggior parte dei decessi per Covid-19 negli Stati Uniti (più del 70\% in alcuni stati) sia tra afroamericani e ispanici (e a New York i tassi di mortalità di ispanici e neri il doppio quelli dei bianchi e degli asiatici americani ${ }^{10}$ ). È una pandemia razzista o l'ennesima dimostrazione di discriminazione di classe, accentuata dalla salute pubblica distrutta dal neoliberismo? Tra queste stesse categorie in

\footnotetext{
${ }^{10}$ Vedi: <https://www.nytimes.com/2020/04/08/nyregion/coronavirus-race-deaths.html>.
} 
Europa vediamo ovviamente molti immigrati regolari e irregolari che continuano a lavorare in particolare nella cosiddetta forza lavoro essenziale dell'economia, come la raccolta di frutta e verdura nelle campagne, i lavori di pulizia, la cura degli anziani, dei bambini, degli adolescenti e dei malati, spesso presso famiglie di lavoratori non confinati in casa o con persone anziane da sole. Tra i tanti paradossi, va segnalato il caso dell'Austria, il paese più chiuso ermeticamente; I'urgente necessità di manodopera agricola per raccogliere asparagi e patate nell'estate 2020 ha costretto il governo ad autorizzare voli charter per il trasporto di lavoratori dalla Romania o da altri paesi dell'Europa orientale. Anche nell'Italia più colpita l'emergere della necessità di manodopera nelle campagne ha spinto il sindacato degli agricoltori a fare pressione sul governo affinché autorizzi l'arrivo di almeno 300.000 immigrati (mentre secondo i sindacati bisognava regolarizzare i circa 600.000 immigrati privi di permesso di soggiorno). Al contrario, nell'aprile 2020 I'Italia ha usato la pandemia come scusa per chiudere i suoi porti alle navi che soccorrevano i migranti in mare ${ }^{11}$.

La situazione degli immigrati rinchiusi nei centri di espulsione è ad alto rischio quanto quella dei detenuti delle normali carceri. Ancora peggiore, tuttavia, è la situazione dei migranti bloccati alle frontiere. Si tratta spesso di persone indebolite, a rischio di malattie respiratorie, polmonite e bronchite, e quindi particolarmente vulnerabili al Covid-19. Sono persone che spesso sono costrette a vivere in spazi affollati e malsani e non ricevono alcun aiuto o materiale medico di prevenzione (dispositivi di protezione individuale (DPI), maschere, ecc.). La situazione non promette nulla di buono per questa popolazione ${ }^{12}$. Al confine di Ventimiglia (Italia), la Francia continua ad espellere i migranti in Italia nonostante il blocco dei confini europei ora giustificato dalla situazione pandemica; nel frattempo le persone espulse sono lasciate con rischi per la salute in condizioni di grave rischio di contagio. Le condizioni dei campi profughi dove migliaia di persone sono stipate in Grecia sono ormai un inferno. Ma nessuno parla più di rifugiati o migranti e in particolare di bambini. Solo poche organizzazioni non governative (ONG) continuano ad essere presenti vicino ai confini. La nave Ocean Viking di Medici Senza Frontiere e SOS Méditerranée è riuscita a portare in Sicilia 274 migranti. Ma subito il leader fascista-razzista Matteo Salvini ne aveva chiesto l'espulsione ${ }^{13}$. Le autorità avevano deciso di metterli in quarantena tutti insieme all'equipaggio, anche se non ci sono prove che i migranti o gli operai della

\footnotetext{
11 Fra altre fonti vedi: < https://www.repubblica.it/cronaca/2020/04/10/news/migranti_porti_chiusi_ per_il_coronavirus_casarini_il_covid_non_puo_essere_il_motivo_-253694321/>.

12 Vedi: $\quad$ https://www.dailymail.co.uk/news/article-8092315/Germany-child-migrants-trappedGreek -islands-prioritise-sick-girls-14.html>.

${ }^{13}$ Vedi: <https://www.ilfattoquotidiano.it/2020/03/21/coronavirus-la-francia-continua-a -respingerei-migranti-a-ventimiglia-ma-con-lemergenza-vengono-abbandonati-senza-precauzioni/5744702/>; e <https://www.notiziegeopolitiche.net/grecia-coronavirus-migranti-e-profughi-dimenticati-nei -campi-di-lesbo-e-moria/>.
} 
Ong siano stati colpiti dal virus. La situazione è drammatica nei campi profughi nelle isole greche. Il primo caso di Covid-19 nel campo profughi dell'isola di Lesbo è stato confermato nel marzo 2020. Ma le autorità di Atene non hanno fornito alcun aiuto concreto, nemmeno ai minori non accompagnati. Secondo I'International Rescue Committee (IRC), Human Rights Watch e il Danish Refugee Council molti bambini non sono adeguatamente registrati e protetti e mancano le procedure di prevenzione delle pandemie. Gli incendi nei campi sono frequenti. Un bambino è morto tra le fiamme divampate in uno dei container dove sono alloggiati i profughi nel campo profughi di Moria. Secondo Stephan Oberreit, capo della missione di Medici Senza Frontiere in Grecia: "Questo incendio arriva solo due mesi dopo l'incendio nel campo di Kara Tepe e solo cinque mesi dopo l'incendio nel campo di Moria nel settembre 2019"14. E recentemente, alcune Ong hanno dovuto ridurre la loro presenza o abbandonare l'area a causa delle violenze contro gli operatori umanitari. Secondo il portavoce dell'UNHCR (Alto Commissario delle Nazioni Unite per i rifugiati) Andrej Mahecic "ci sono più di 36.000 richiedenti asilo che soggiornano in centri di accoglienza in cinque isole, originariamente progettati per 5.400 persone $^{\prime 15}$. La ONG Medici Senza Frontiere teme che l'epidemia di Covid-19 si tradurrà in una nuova ingiustificata ansia pubblica nei confronti di coloro che sono stati salvati in mare e fungerà da "scusa per impedire a Ocean Viking di riprendere la sua opera di controllo nel Mediterraneo centrale"16.

La chiusura quasi totale delle frontiere da parte di tutti gli stati non fa che accentuare il divieto di migrare. Pertanto, ancora una volta, la nozione di tanatopolitica è cruciale per comprendere questa situazione - vale a dire per far morire queste persone senza diritti, viste e concepite come "umanità in eccesso" (un concetto che è stato utilizzato principalmente nel campo degli studi sull'olocausto, nella crisi umanitaria e negli studi sui campi profughi). Così, la logica cinica e brutale delle frontiere del neoliberismo economico globalizzato diventa, con tutti questi esempi dettagliati, ancora più concreta nella tragedia umanitaria della migrazione come illustrazione chiave di ciò che accade ai confini globali. L'esempio più tragico è dato nel mese di dicembre 2020 e gennaio 2021 da quanto succede nei Balcani. Il 23 dicembre il campo profughi di Lipa è stato chiuso. Durante lo sgombero un incendio - probabilmente provocato apposta dalla polizia - ha distrutto la struttura. Centinaia di profughi sono rimasti al gelo e sotto la neve. A causa delle proteste della popolazione locale è stato impossibile spostarli in altri campi. Ora a Lipa l'esercito sta allestendo le tende, ma manca

\footnotetext{
${ }^{14}$ Vedi: <https://www.notiziegeopolitiche.net/grecia-coronavirus-migranti-e-profughi-dimenticatinei -campi-di-lesbo-e-moria/>.

${ }^{15}$ Vedi: <https://www.notiziegeopolitiche.net/grecia-coronavirus-migranti-e-profughi-dimenticatinei -campi-di-lesbo-e-moria/>.

${ }^{16} \mathrm{Vedi}><$ https://www.notiziegeopolitiche.net/grecia-coronavirus-migranti-e-profughi-dimenticatinei -campi-di-lesbo-e-moria/ $>$.
} 
tutto: servizi, elettricità, acqua. "Lasciare i migranti a Lipa è la peggiore delle soluzioni possibili", spiega Daniele Bombardi, coordinatore Caritas Italiana del Sud Est Europa, "siamo davanti ad una catastrofe umanitaria". La situazione si è ancor più aggravata con altri i respingimenti violenti dalla Croazia ${ }^{17}$.

I migranti fanno la scommessa disperata, il game, come dicono loro per indicare il passaggio tra il confine bosniaco e quello croato, ma vengono scoperti dalla polizia croata, picchiati, torturati, derubati e poi rispediti indietro ${ }^{18}$.

\section{Conclusioni}

Il cambiamento nel paradigma della migrazione e delle frontiere non può essere compreso se non si tiene pienamente conto di due aspetti principali: 1) le conseguenze del trionfo del liberismo globalizzato, 2) la paura dei dominanti rispetto all'aumento della popolazione mondiale che considerano incontrollabile e che pensano che si sovrapponga al cosiddetto cambiamento climatico. Questi fatti hanno trasformato radicalmente la struttura economica, sociale, culturale e politica del mondo a livello locale e globale; a partire dagli anni Ottanta, siamo entrati in una nuova era (quella del capitalocene piuttosto che dell'antropocene ${ }^{19}$ ). L'era precedente è stata segnata dallo sviluppo capitalista più o meno inquadrato dagli stati-nazione con un ampio ricorso alla migrazione interna e internazionale. Tutte le potenze economiche, militari e politiche, guidate dagli Stati Uniti, hanno sviluppato le loro società grazie a queste migrazioni, integrandole, assimilandole o adattandole ad essere lavoratori docili e anche cittadini servili e rigettando chi non sottostava a queste condizioni. Questo modello s'è imposto durante il periodo della biopolitica: integrazione e assimilazione della maggioranza degli immigrati e rigetto del "cattivo immigrato", cioè di coloro che non possono o non vogliono diventare lavoratori docili cittadini (cfr. Preciado, 2020). Oggi, lo sviluppo economico ha molto meno bisogno di lavoratori stabili rispetto a prima degli anni '70, ma di lavoro instabile, segmentato e flessibile. Si richiede manodopera "usa e getta" o anche schiavizzata nel cuore delle stesse grandi città del mondo ricco come nelle campagne. L'abbondante disponibilità di manodopera immigrata insieme all'erosione delle protezioni dei lavoratori offre ai datori di lavoro la possibilità di abbassare i salari e peggiorare le condizioni di lavoro, a volte schiavizzando. La fine dell'inserimento tradizionale dei migranti

\footnotetext{
17 Vedi: <https://www.avvenire.it/attualita/pagine/rotta-balcanica-respingimenti-a-catena-colpitianche-i-disabili $>$.

${ }^{18}$ Vedi: <http://www.vita.it/it/article/2021/01/04/bosnia-e-quei-migranti-che-sta-condannando-amorte/157883/>; Gostoli, Ylenia. Viaggio senza fine sulla rotta dei Balcani. The New Humanitarian, Svizzera, 11.12.2020, <https://www.internazionale.it/notizie/ylenia-gostoli/2020/12/11/migrantirotta-balcani>; in inglese qui: < https://www.thenewhumanitarian.org/news-feature/2020/11/17/ europe-italy-bosnia-slovenia-migration-pushbacks-expulsion $>$.

${ }^{19}$ Vedi: <http://effimera.org/negazionismo-scetticismo-o-resistenze-dove-va-lecologia-politica-dituri-palidda/ $>$.
} 
è drammaticamente illustrata dalla precaria sopravvivenza dei migranti nei paesi ricchi (compresi gli Emirati Arabi e l'Arabia Saudita).

La tendenza a scegliere l'opzione di far morire i migranti (la tanatopolitica) è stata adottata da tutti i paesi ricchi; la pseudo democrazia occidentale è sempre più eterogenesi delle promesse del secondo dopoguerra. La violenza razzista, fascista e sessista è abitualmente praticata non solo da quella parte delle polizie che ne abusa ma anche da una parte della popolazione che vuole trarre profitto dalla negazione dei diritti degli immigrati o che ha paura del declino della supremazia dei bianchi (vedi il caso dei seguaci dei Trump, Bolsonaro, Erdogan, Orban, Salvini o Boris Johnson e anche Macron).

Approfittando della pandemia da Sars-Covid 19 tutti gli Stati dominanti hanno accentuato la chiusura delle frontiere e la persecuzione dei migranti. La pandemia è sfruttata per erodere sia le possibilità e occasione di agire politico, sia per accentuare le azioni repressive, sia per rendere impossibile anche le attività di solidarietà e di soccorso da parte delle ONG. Tutto ciò ancora di più in nome della crisi economica derivante dalla pandemia. Nel frattempo le multinazionali hanno scatenato la loro speculazione per i vaccini che quindi non saranno disponibili per la popolazione povera di tutti i paesi del mondo. Si produrrà così un ulteriore aggravamento delle condizioni di vita delle popolazioni che già sono allo stremo e non potranno migrare.

L'ultima prova di cosa è diventata la guerra alle migrazioni è anche in un articolo del quotidiano Le Monde ${ }^{20}$. In esso si rivela che il canale pubblico tedesco ZDF con la collaborazione dell'ONG Corporate Europe Observatory (CEO), hanno svolto un'indagine sui legami tra l'Agenzia europea della Guardia-frontiere e l' industria della sorveglianza e degli armamenti. Decine di documenti, a cui ha avuto accesso anche Le Monde, dimostrano violazioni delle regole delle istituzioni europee sul lobbismo, una mancanza di trasparenza e una quasi totale assenza di preoccupazione per il rispetto dei diritti umani. Come da anni documenta anche Statewatch, Frontex è di fatto diventato una sorta di associazione di funzionari che flirtano anche con mercenari e bande criminali (come quelle libiche) che pratica la tanatopolitica (il lasciar morire). Di fatto i soldi dei cittadini contribuenti europei sono spesi per un crimine contro l'umanità. E' quindi auspicabile una mobilitazione dei cittadini europei per la soppressione di questa Agenzia che sinora allegramente viola quei diritti umani che l'Unione Europea dice di difendere.

L'unica soluzione sostenibile sarebbe l'equa distribuzione della ricchezza, l'abolizione dei profitti, l'abolizione di tutte le attività devastanti che causano disastri sanitari e ambientali e la maggior parte dei decessi per malattie dovute a contaminazioni tossiche e condizioni di lavoro e di vita insostenibili - cioè un

\footnotetext{
${ }^{20}$ Vedi: <https://www.lemonde.fr/international/article/2021/02/05/nouvelles-accusations-contre-
} frontex-l-agence-europeenne-des-gardes-frontieres_6068963_3210.html >. 
drammatico aumento della insicurezza degli umani e di tutto il mondo animale e vegetale.

Tuttavia, anche se la situazione tende a peggiorare nel breve e medio termine, la migrazione continuerà inesorabilmente e, nonostante gli ostacoli, i migranti riusciranno a integrarsi almeno parzialmente nei paesi di arrivo / accoglienza. La resistenza dei migranti, così come di altre persone vittime del dominio liberista e delle sue scelte è una questione di sopravvivenza e l'espressione dell'aspirazione mai sopita all'emancipazione economica, sociale, culturale e politica.

\section{Bibliografia}

ARADAU, Claudia; TAZZIOLI, Martina. Biopolitics multiple: Migration, extraction, subtraction. Millennium, v. 48, n. 2, p. 198-220, 2020. Disponibile su: <http:// research.gold.ac.uk/27738/1/Biopolitics\%20multiple\%20Accepted\%20manuscript. pdf $>$.

BAUMAN, Sigmund. Wasted Lives: Modernity and Its Outcasts. Cambridge: Polity, 2003.

CUTTITTA, Paolo. Delocalization, humanitarianism, and human rights: The Mediterranean border between exclusion and inclusion. 2017. Disponibile su: $<$ https://onlinelibrary.wiley.com/doi/full/10.1111/anti.12337>.

DAL LAGO, Alessandro; PALIDDA, Salvatore (eds.). Conflict, Security and the Reshaping of Society: The Civilisation of War. London: Routledge, 2010. Disponibile su: <http://www.oapen.org/search?identifier $=391032>$.

FOUCAULT, Michel. Sécurité, Territoire, Population: Cours au Collège de France, 1977-1978. Paris: Hautes Etudes-Seuil-Gallimard, 2004.

FOUCAULT, Michel. Naissance de la biopolitique: Cours au Collège de France, 19781979. Paris: Hautes Etudes-Seuil-Gallimard, 2005.

GROS, Frédéric. Désastre humanitaire et sécurité humaine. Le troisième âge de la sécurité. 2008. Disponibile su: <https://esprit.presse.fr/article/gros-frederic/desastrehumanitaire-et-securite-humaine-le-troisieme-age-de-la-securite-14470>.

GUILD, Elspeth; BIGO, Didier (eds.). Controlling Frontiers: Free Movement into and within Europe. London: Routledge, 2005.

HEYMAN, Josiah; RIBAS-MATEOS, Natalia. Borders of Wealth and Poverty: Ideas Stimulated by Comparing the Mediterranean and U.S.-Mexico Borders. Archivio antropologico mediterraneo, v. 21, n. 2, 2019. DOI: <https://doi.org/10.4000/ aam.2019>.

MARTINI, Francesca; PALIDDA, Salvatore. Continuità e mutamenti delle migrazioni nel confine tra l'Italia e la Francia. Altreitalie, v. 56, p. 117-129, 2018. Disponibile su: <https://www.altreitalie.it/pubblicazioni/ rivista/n-56/acquista-versione-digitale/ continuita-e-mutamenti-delle-migrazioni-nel-confine-tra- litalia-e-la-francia.kl>.

MILLER, Todd. Storming the Gate: Climate Change, Migration, and Homeland Security. San Francisco: City Lights, 2017. 
PALIDDA, Salvatore (ed.). Razzismo democratico. La persecuzione degli stranieri in Europa. Milano: Agenzia X, 2009.

PALIDDA, Salvatore (ed.). Resistenze ai disastri sanitari-ambientali ed economici nel Mediterraneo. Roma: Derive\&Approdi, 2018a.

PALIDDA, Salvatore. La guerre aux migrations ou la subsomption de tous les désastres de la derive néo-libériste: le fait politique total du XXI siècle. 2018b. Disponibile su: $<$ https://www.academia.edu/37936402/La_guerre_aux_migrations_ou_la_subsom ption_de_tous_les_désastres_de_la_dérive_néo_libériste_le_fait_politique_total_ du_XXI_siècle>.

PALIDDA, Salvatore. Negazionismo, scetticismo o resistenze: dove va l'ecologia politica?. 2019. Disponibile su: < http://effimera.org/negazionismo-scetticismo-oresistenze-dove-va-lecologia-politica-di-turi-palidda $>$.

PERKINS, John. Confessions of an Economic Hit Man. London: Ebury Press, 2005.

PRECIADO, Paul B. Les leçons du virus. Mediapart, 11.04.2020. Disponibile su: $<$ https://www.mediapart.fr/journal/culture-idees/110420/les-lecons-du-virus >.

RIBAS-MATEOS, Natalia. Border Shifts. New Mobilities in Europe and Beyond. New York: Palgrave Macmillan, 2015.

SASSEN, Saskia. Globalization and Its Discontents: Essays on the New Mobility of People and Money. New York: New Press, 1999.

SASSEN, Saskia. Territory, Authority, Rights: From Medieval to Global Assemblages. Princeton: Princeton University Press, 2008.

SASSEN, Saskia. Expulsions: Brutality and Complexity in the Global Economy. Cambridge, MA: Harvard University Press, 2014.

SAYAD, Abdemalek. La doppia assenza. Milano: Cortina, 2000.

SQUIRE, Vicki. Governing migration through death in Europe and the US: Identification, burial and the crisis of modern humanism. European Journal of International Relations, Settembre, 2016. Disponibile su: <https://journals.sagepub.com/doi/ full/10.1177/1354066116668662>. 